\title{
Langues, littératures et forces antinomiques. Le cas de la Belgique
}

Languages, literatures and antinomic forces: the case of Belgium

Paul Dirkx

\section{Q OpenEdition}

\section{Journals}

Édition électronique

URL : https://journals.openedition.org/lbl/565

DOI : $10.4000 / / b l .565$

ISSN : 2727-9383

Éditeur

Université de Bretagne Occidentale - UBO

\section{Édition imprimée}

Date de publication : 30 décembre 2019

Pagination : 145-159

ISBN : 979-10-92331-49-3

ISSN : 1270-2412

\section{Référence électronique}

Paul Dirkx, "Langues, littératures et forces antinomiques. Le cas de la Belgique », La Bretagne Linguistique [En ligne], 23 | 2019, mis en ligne le 01 mai 2020, consulté le 05 mai 2021. URL : http:// journals.openedition.org/lbl/565; DOI : https://doi.org/10.4000/lbl.565

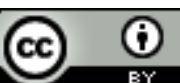

La Bretagne Linguistique est mise à disposition selon les termes de la Licence Creative Commons Attribution 4.0 International. 


\title{
Langues, littératures et forces antinomiques. Le cas de la Belgique
}

\author{
PAUL DIRKX*
}

\begin{abstract}
Cet article entend rouvrir le dossier des rapports entre l'organisation politique et la configuration littéraire en Belgique. Il est susceptible d'intéresser les spécialistes des "minorités culturelles» en France, notamment en Bretagne, même s'il n'y fera guère allusion. Notre point de départ sera un facteur qui se voit généralement traité rapidement, voire négligé quand il s'agit de langue et de littérature en Belgique, à savoir les conditions de genèse de l'État. Ces conditions peuvent être éclairées utilement à l'aide du concept d'antinomie, qui sera au centre d'une première partie. Ensuite seront abordés les effets de l'antinomie sur les institutions belges en général, sur les langues en particulier et, enfin, sur les productions littéraires.
\end{abstract}

\section{Antinomie et État belge}

Il convient d'abord de préciser ce que nous entendons par «État». En nous inspirant d'Émile Durkheim, de Max Weber et de Pierre Bourdieu ${ }^{1}$, nous dirions qu'il s'agit essentiellement d'une

\footnotetext{
* Maitre de conférences (HDR) en littérature, Écritures (EA 3943), Université de Lorraine.

*1. Émile DURKHEIM, Les formes élémentaires de la vie religieuse. Le système totémique en Australie, Paris, Presses Universitaires de France, coll. "Quadrige», 2008 [1912] ; Max Weber, Économie et société. Traduit par Julien Freund [e. a.], Paris, Plon, coll. "Recherches en sciences humaines», 1971 ; Max WEBER, La domination. Traduit de l'allemand par Isabelle Kalinowski, Paris, La Découverte, coll. «Poche», 2015 ;
} 
organisation capable de définir ${ }^{2}$ et de faire accepter par tous les corps individuels et collectifs installés sur un territoire donné, lesquels s'en trouvent ainsi constitués et institués en "État», un principe unique et donc contraignant de vivre ensemble, en l'universalisant comme une condition existentielle exclusive.

En grec, on désigne un tel principe de vision et de division par le mot "nomos», dont Pierre Bourdieu a fait un concept dans sa théorie générale des champs ${ }^{3}$. Dans le cas de l'État français, le nomos, véritable impératif existentiel catégorique, est : «ici, sur ce territoire, il y a lieu d'être français", le mot «lieu» exprimant bien la territorialité de la raison d'être sacrée. Pour l'Allemagne, c'est «ici, il y a lieu d'être allemand", ou, ailleurs encore, "être néerlandais», "être espagnol», etc. L'activité fondatrice et vitale de l'État français est donc de produire des corps «français» qui se vivent comme tels à l'aune du nomos étatique. Ce qui fonderait donc une organisation étatique, ce serait sa capacité à amener le corps social qu'elle enferme littéralement à l'intérieur de ses frontières à se soumettre de lui-même au nomos qu'il éprouverait de ce fait comme le sien propre («identité»). L'État serait ce qui est à même d'enfermer deux fois une masse territorialisée de corps, à savoir sur un mode inséparablement physique et symbolique.

Cette capacité à inciter les corps à se soumettre durablement d'euxmêmes (en grec, «autos») au nomos peut être appelée autonomie. Elle est pour ainsi dire le contraire de l'indépendance : elle fonde un coup

Pierre Bourdieu, Sur l'État. Cours au Collège de France (1989-1992), Paris, Raisons d'agir, coll. «Cours et travaux», 2013.

2. Le terme "organisation" devrait être à son tour précisé, ce qui nécessiterait de nouveaux compléments à notre caractérisation, compléments qui eux-mêmes demanderaient de nouvelles spécifications, etc. C'est pourquoi donner une définition de la notion d'État (ou de toute autre notion), au sens de dé-finir, dé-limiter, déterminer, est une vaine entreprise. Outre qu'il est un objet "presque impensable» (ibidem, p. 13), l'État est indéfinissable, alors même qu'il a pour monomanies la production et l'imposition de définitions (à travers l'École, etc.), à commencer par la sienne propre.

3. Cf. Pierre Bourdieu, Propos sur le champ politique. Avec une introduction de Philippe Fritsch, Lyon, Presses Universitaires de Lyon, 2000, surtout p. 51-64. Le sociologue a élaboré ce concept de manière systématique dans ses écrits sur le champ littéraire (id., Les règles de l'art. Genèse et structure du champ littéraire, Paris, Seuil, coll. «Libre examen», 1992, p. 310-321). 
de force permanent, un coup d'État à échelle individuelle, perpétré plus ou moins spontanément et à longueur de vie par chaque corps sur lui-même, et qui signe la capacité définitoire qui fonde le pouvoir de l'État. L'histoire d'un État peut alors être analysée en partie comme un processus d'autonomisation.

Ce processus semble impliquer deux phases. Il consiste d'abord en une émancipation progressive vis-à-vis des structures de pouvoir concurrentes, externes, en premier lieu le pouvoir religieux. Mais bien vite, la question devenant une question de vie ou de mort, cette émancipation en vient à produire une sensation de solidarité et une mémoire collective issues notamment des diverses luttes d'émancipation. Autrement dit, elle génère une cohésion interne qui finit par prendre une forme absolue, ontologique, celle d'un nomos («ici, il y a lieu d'être $\mathrm{X}$ ou $\mathrm{Y} »)$. Ce nomos va ensuite s'universaliser à tout le territoire pour devenir un jour, le cas échéant, le fondement d'un État. Ainsi, «être français» exprime cet esprit de corps étatique, esprit de corps qui devient une réalité indistinctement matérielle et immatérielle en s'incarnant dans d'innombrables institutions et d'innombrables expressions, telles que «peuple français», «identité nationale», "esprit républicain», etc. ${ }^{4}$.

Venons-en alors au cas de l'État en Belgique. Ici, le processus d'autonomisation, qui remonte au $\mathrm{XVI}^{\mathrm{e}}$ siècle ${ }^{5}$, s'est déroulé dans des conditions non pas artificielles - aucun État, on l'a vu, n'est naturel -, mais pour le moins précaires. Le territoire, tout d'abord, est non seulement exigu, mais pris en tenaille entre les deux principales puissances du continent, la future France et la future Allemagne. Il semble permis de dire que celles-ci ont passé leur temps, depuis le IX siècle et le traité de Verdun de 843, à digérer la Lotharingie, cet empire du milieu dont la Lorraine, l'Alsace, le Nord-Pas-de-Calais, le Luxembourg, la Hollande et la Belgique actuels apparaissent comme autant de résidus. Les «Pays-

4. Pour un exemple n'ayant pas (encore) abouti à un État, lire Catherine BERTHO, "L'invention de la Bretagne. Genèse sociale d'un stéréotype», Actes de la recherche en sciences sociales, $\mathrm{n}^{\circ} 35$, novembre 1980 , p. 45-62.

5. Jean STENGERS, Histoire du sentiment national en Belgique des origines à 1918. Tome 1 : Les racines de la Belgique. Jusqu'à la Révolution de 1830, Bruxelles, Racine, 2000 ; Jean STENGERS et Éliane GUBIN, Histoire du sentiment national en Belgique des origines à 1918. Tome 2 : Le grand siècle de la nationalité belge. De 1830 à 1918, Bruxelles, Racine, 2002. 
Bas» ("Lage Landen» en néerlandais, "Belgica» en latin) qui, dès avant l'apogée du duché de Bourgogne au XV $\mathrm{XV}^{\mathrm{e}}$ siècle, correspondaient à peu près à l'actuel Benelux, se sont scindés en deux à la suite des guerres de religion vers la fin du XVI siècle. En outre, l'État belge en tant que tel a émergé assez tard, en 1831, qui plus est sur une scène européenne où l'enjeu national était extrêmement inflammable ${ }^{6}$.

Voilà une situation de départ bien précaire qui, a priori, n'est guère propice à l'intériorisation ou, plus exactement, à l'incorporation massive d'un nomos belge pourtant plus que latent ${ }^{7}$, nomos qu'Ernest Renan appelait "une profonde raison d'être» que seul «un plébiscite de tous les jours" peut transmuer en nation ${ }^{8}$. Néanmoins, après une première tentative avortée en 1790 , puis à la suite d'une révolution populaire en août-septembre 1830, un État souverain est mis sur pied qui, malgré tout, parvient à donner corps à une raison d'être spécifique à la Belgique. Mais, avec le temps, cet État s'est de moins en moins réclamé, de manière positive, de ses «racines", pourtant assez anciennes, et de plus en plus, de manière négative, de ses différences d'avec ses voisins. Il s'est donc approprié le nomos local, mais en lui donnant une forme toujours plus différentielle et un horizon toujours plus extraterritorial, désincarné : «ici, on n’est pas français, on n'est pas allemand, ni néerlandais, ni espagnol, ni autrichien». Tout laisse à penser que, à force de se targuer d'une originalité ni française, ni allemande, etc., à force de s'afficher comme le carrefour «des deux grandes civilisations» européennes, "la latine et la germanique ${ }^{9}$ », cet État a moins consolidé son propre nomos qu'il n'a renforcé la foi dans le nomos de ses concurrents limitrophes. Pareille indécision est a priori intenable, s'agissant d'un impératif catégorique qui ne souffre aucune concession et qui n'est donc compatible avec aucun autre.

6. Anne-Marie THIESSE, La création des identités nationales. Europe XVIIT-XXe siècle, Paris, Seuil, coll. «Points», 2001 [1999].

7. J. STENGERS, $o p$. cit.

8. Ernest RENAN, "Qu'est-ce qu'une nation ? Conférence faite en Sorbonne, le 11 mars 1882 », Discours et conférences, Paris, Calmann-Lévy, 1887, p. 277-310, p. 287 et 307. Cf. "Le sentiment national n'est spontané que lorsqu'il a été parfaitement intériorisé» (A.-M. THIESSE, op. cit., p. 14).

9. Ce poncif n'aura pas besoin d'être théorisé par l'écrivain-avocat Edmond Picard à partir de 1897 pour connaître un succès qui ne s'est que rarement démenti. 
Cette incompatibilité, cette cohabitation conflictuelle de deux principes exclusifs, nous proposons de l'appeler antinomie ${ }^{10}$. À resémantiser un mot devenu banal, ce concept permet de mieux penser ensemble l'autonomie, motivée par cette matrice fondatrice qu'est le nomos propre, et l'hétéronomie, c'est-à-dire l'adhésion au nomos d'un autre («heteros» en grec). Le concept d'antinomie favorise une prise en compte conjointe des tendances indissociablement autonomes et hétéronomes qui (dé)structurent un corps humain, qu'il soit individuel ou collectif, par exemple un État. Il incite ainsi à reconsidérer à nouveaux frais certains aspects de l'histoire de l'État en Belgique depuis 1831. Cette histoire peut se lire comme l'enchaînement d'un certain nombre d'effets de l'antinomie étatique sur toute une société.

\section{Effets de l'antinomie étatique sur les institutions belges en général}

Il faut s'arrêter aux effets de l'antinomie sur les institutions en général (politiques, sociales, économiques, etc.). Nous avons essayé de décrire ailleurs ${ }^{11}$ comment le jeune État belge s'est efforcé d'exorciser ses démons antinomiques et de neutraliser sa double attirance pour la France et pour l'Allemagne. À cet effet, il a introduit simultanément dans ses rouages, d'une part, une dose de propriétés relevant du nomos français et de ce que ce mot "français » impliquait à l'époque en matière de progressisme libéral, et, d'autre part, une dose relevant du nomos allemand et de son esprit d'Ancien Régime. Cet alliage forme par exemple la trame de la Constitution belge qui, fondée sur la cohabitation d'un pouvoir monarchique fort et d'un système bicaméral, est saluée internationalement comme un modèle de protection des libertés individuelles et collectives.

10. Paul DirkX, Les "amis belges». Presse littéraire et franco-universalisme, Rennes, Presses Universitaires de Rennes, coll. "Interférences», 2006, p. 28-31. Voir la notice que Pierre Halen consacre à ce nouveau concept dans Le lexique socius [A. Glinoer et D. Saint-Amand (dirs.), URL : http://ressources-socius.info/index.php/lexique/21lexique/50-antinomie, consulté le 29 septembre 2019].

11. Paul DirkX, La concurrence ethnique. La Belgique, l'Europe et le néolibéralisme, Bellecombe-en-Bauges, Le Croquant, coll. "Savoir / agir», 2012 ; Paul DIRKX, «Pour finir. Une illusion si mal fondée ?», dans A. von Busekist (dir.), Singulière Belgique, Paris, Fayard, coll. «Les grandes études internationales», 2012, p. 229-242. 
Or, comme en témoigne amplement l'histoire des $\mathrm{XX}^{\mathrm{e}}$ et $\mathrm{XXI}^{\mathrm{e}}$ siècles, ce savant dosage n'a pas su juguler les tensions antinomiques, d'autant moins que Paris et Berlin en ont régulièrement tiré profit pour s'ingérer plus ou moins directement dans les affaires belges. Cherchant à compenser cette fragilité structurelle, l'État belge met tout en œuvre pour se concilier les puissances, par exemple en respectant scrupuleusement la neutralité diplomatique qu'elles lui ont imposée. Sur le plan intérieur, il prend l'habitude de ménager tous les acteurs sociaux, politiques, économiques, etc. en privilégiant le compromis pour le compromis (dit «à la belge»). Il va jusqu'à se livrer à une sorte de délégation de pouvoir, de sous-traitance d'un nombre croissant de ses prérogatives : sous-traitance de certains actes politiques aux deux, puis aux trois partis politiques «de gouvernement» (catholique, libéral et socialiste) ; sous-traitance de certains services publics aux "piliers", c'est-à-dire aux réseaux d'institutions (syndicats, institutions de santé, d'éducation, de presse, etc.) qui sont associés à ces partis; sous-traitance d'opérations et de services publics à des entreprises privées, par exemple la colonisation du Congo confiée pendant tout un temps à la personne du roi Léopold II en tant que personne privée.

En cédant de la sorte des parts importantes de son pouvoir, l'État n'a fait qu'accroître son instabilité relative. En effet, progressivement, les partis, les piliers, etc. tendent à se comporter toujours davantage comme autant d'États dans l'État qui contestent son crédit et plus précisément son monopole de l'autonomie - voire, comme nous le verrons plus loin, le bien-fondé du nomos «être belge»-, en l'orientant le plus souvent vers l'hétéronomie économique. Hétéronomie économique pour ainsi dire assumée : l'État belge, qui a été édifié par des fractions de la bourgeoisie solidement adossées aux marchés financiers et à un tissu industriel de premier plan, se montre nettement enclin à prioriser les logiques économiques, en croyant celles-ci compatibles avec les siennes propres et en conditionnant les intérêts de la collectivité nationale à ceux d'une partie de celle-ci. Cette stratégie se révélera des plus payante, puisque, jusqu'en 1914, la Belgique se situera parmi les cinq principales puissances économiques du monde. Mais les effets de cette hétéronomisation sur le crédit non pas financier, mais politique de l'État furent moins positifs, dans la mesure où l'externalisation des pouvoirs n'a pas aidé à compenser, pour peu que ce fût le but, sa vulnérabilité liée 
à son antinomie originelle, au contraire.

L'exemple à la fois le plus patent et le plus inaperçu du caractère contre-productif de cette stratégie d'autoconservation - l'exemple le plus permanent aussi, quoique jamais analysé comme tel - est la gestion par l'État de la langue et de la culture.

\section{Effets de l'antinomie politique en matière linguistique et culturelle}

Inspirée par le libéralisme romantique de l'époque, la Constitution belge prévoit que «l'emploi des langues usitées en Belgique est facultatif ${ }^{12}$ ». Mais dans les faits, c'est la langue française qui devient rapidement le mode d'expression unique de l'appareil d'État. Non seulement le français est propre aux groupes sociaux arrivés au pouvoir (en Belgique comme dans de nombreuses autres parties de l'Europe), mais il est la langue opérationnelle dans l'essentiel des administrations du royaume. Le néerlandais standard, outre qu'il n'est guère compris des locuteurs des dialectes du néerlandais (brabançons, flamands, limbourgeois) et encore moins des autres populations, est l'idiome plus ou moins abhorré, y compris dans le Nord, de l'occupant hollandais qui vient d'être chassé du territoire pour avoir, entre autres, essayé d'imposer sa langue à des échelons administratifs ou dans des régions où elle n'avait jamais eu cours.

Les fondateurs de l'État belge ont sans doute voulu faire de leur langue un élément d'unification ${ }^{13}$. Mais leur pari était pour le moins risqué : en faisant du français la langue légitime quasi exclusive, ils se sont mis objectivement au service d'une variante locale du modèle "universaliste» français qui, pour sa part, avait eu trois siècles pour s'enraciner dans un territoire dix-sept fois plus grand que la Belgique, à travers la triade langue française - culture française - nation française ${ }^{14}$.

12. Constitution belge de 1831, article 23 (article 30 dans la version en vigueur en 2020). Sur la question des langues en Belgique, voir Albert VERDOODT, Les problèmes des groupes linguistiques en Belgique, Louvain, Peeters, 1977 ; Astrid VON BUSEKIST, La Belgique. Politique des langues et construction de l'État, de 1780 à nos jours, Paris Bruxelles, Duculot, 1998 ; Els Witte et Harry Van Velthoven, Strijden om taal. De Belgische taalkwestie in historisch perspectief, Kapellen, Pelckmans, 2010.

13. A. VON BUSEKIST, op. cit., p. 63-64.

14. Cf. Joseph HANSE, "Littérature, nation et langue», Bulletin de l'Académie 
Le qualificatif «français" ayant dans ce schème une portée beaucoup moins universelle que nationale et l'«universalisme français» étant en réalité un franco-universalisme ${ }^{15}$, la politique linguistique de l'État belge a fait pencher l'image qu'il donne de lui-même du côté du nomos français, compromettant ainsi gravement, et jusqu'à nos jours, son fragile équilibre antinomique. En outre, la variante belge de l'«universalisme français» fut implantée dans un pays où la langue n'avait jamais joué de rôle sociopolitique majeur. Elle y était encore plus inadaptée que chez les voisins du Sud qui, en 1831, n'étaient toujours pas une majorité à utiliser la langue française ${ }^{16}$. Dans les provinces belges du nord, la langue française ne réussit à rivaliser avec les dialectes (toujours vivaces de nos jours) que dans une partie de la vie professionnelle, surtout en zone urbaine. Mais elle eut à compter aussi au sud, surtout en milieu rural, et jusqu'à la Première Guerre, avec la domination nette des dialectes wallons, lorrains et picards ${ }^{17}$.

Dans la moitié nord, cet «universalisme français » à la belge donne naissance, dès 1840 , à un mouvement de réaction dit «flamingant", qui promeut le schéma allemand, herderien, «une langue - une culture - un peuple». Sa devise est "De tael is gansch het volk»: la langue est le peuple même. Après avoir "encommissionné» («sous-traité» à une commission) les "griefs flamands" pendant plusieurs décennies, l'État se met timidement à rendre obligatoire le néerlandais par le biais d'une première "loi linguistique» en 1873. Mais il met ainsi le doigt dans un engrenage périlleux, car pareille obligation implique la

Royale de Langue et de Littérature Françaises, tome 42, n 2, 1964, p. 93-114; Henri Meschonnic, De la langue française. Essai sur une clarté obscure, Paris, Hachette, coll. "Pluriel», 1997 ; Dominique COMBE, "L'invention des littératures nationales en langue française au XIX ${ }^{\mathrm{e}}$ siècle. Langue, nation, littérature», dans Sarga Moussa (dir.), Le XIXe siècle et ses langues. Ve Congrès de la Société des Études Romantiques et Dix-neuviémistes, 2013, URL : http://etudes-romantiques.ish-lyon.cnrs.fr/langues. html (consulté le 15 septembre 2019).

15. P. DIRKX, Les "amis belges", op. cit.

16. Eugen WEBER, La fin des terroirs. La modernisation de la France rurale 1870-1914. Traduit de l'anglais par Antoine Berman et Bernard Géniès, Paris, Librairie Arthème Fayard / Éditions Recherches, 1983, p. 108-111.

17. D. Blampain, A. Goosse, J.-M. Klinkenberg et M. Wilmet (dirs.), Le français en Belgique. Une langue, une communauté, Bruxelles, Duculot / Communauté française de Belgique, 1997, p. 230-231. 
délimitation d'un territoire d'application, donc l'établissement d'une "frontière linguistique ${ }^{18}$ ». L'État belge et, de par son fonctionnement antinomique, le système des États européens donnent ainsi naissance à deux entités jusque-là inexistantes : d'un côté de la "frontière», la Flandre (qui n’a que de lointains rapports avec le comté médiéval de Flandre, «les Flandres» désignant ensuite le plus souvent la Belgique entière, à l'exclusion de la principauté de Liège) et, de l'autre côté, la Wallonie, mot qui ne commence à se répandre qu'en 1886, lorsqu'est créée la revue symboliste liégeoise La Wallonie.

Par la même occasion, l'État belge déclenche deux processus d'autonomisation étatique en son sein. Pris au piège de son indétermination antinomique en matière «linguistique ${ }^{19}$ ", il n'envisagera jamais d'autre issue que celle qui consiste à reconnaitre toujours un peu davantage l'existence simultanée de deux peuples jusquelà inexistants auxquels il ne songera qu'à céder, comme il en a pris l'habitude dans d'autres domaines, un nombre croissant de leviers institutionnels au niveau culturel - niveau sous-estimé par lui, et que le libéralisme ambiant des élites lui conseille de laisser en "liberté». L'écart entre "français» et "germanique» se creusant mécaniquement, en-deçà de la ligne de démarcation linguistique en faveur du premier terme et, au-delà, en faveur du second, l'État belge s'obligera ainsi luimême à supporter l'exacerbation graduelle de ses tensions antinomiques internes. En 1970, il prendra le parti de se dessaisir de ce qui était devenu bien davantage qu'une "question linguistique», en sous-traitant les compétences devenues sources de discorde «entre Flamands et Wallons» à de nouvelles institutions créées à cet effet : trois "Communautés»

18. Pascal DeLWIT, La vie politique en Belgique de 1830 à nos jours, Bruxelles, Éditions de l'Université de Bruxelles, coll. UBlire, 2010, p. 42-43. Sur le caractère fictif de la notion de "frontière linguistique» d'un point de vue empirique, lire Guy VANDE PUTTE, Belgica Creola. Le contact des langues en périphérie bruxelloise. L'exemple d'Overijse, Bruxelles - Anvers, EPO, 1999.

19. La lenteur dont fait preuve l'État belge, à l'instar de sa gestion des injustices sociales et coloniales, ainsi que la permanence du français comme première langue de l'État jusque dans les années trente du siècle suivant, ne font qu'alimenter la frustration des «flamingants». L'Allemagne va sciemment attiser cette frustration pendant au moins trois décennies, entre 1914 et 1944 . De cette période, le "mouvement flamand" sortira déterminé à en finir avec l'État belge «fransquillon», puis avec l'État belge tout court. 
(française de Belgique, flamande et germanophone de Belgique ${ }^{20}$ ) pour ce qui est des affaires "personnalisables» (culturelles), et trois «Régions" (Région wallonne, Région flamande et Région de Bruxelles-Capitale) pour les dossiers socio-économiques régionaux.

Ainsi, après s'être taillé une place de tête dans le "concert des nations" pendant un siècle, le pays ne cessera plus de se scinder en deux sur la base de cette "frontière» qui, régie par plusieurs lois de l'entre-deux-guerres, puis fixée une fois pour toutes par des lois spéciales en 1962 et 1963, constituera la colonne vertébrale d'une des entreprises de fédéralisation les plus poussées au monde. Aujourd'hui, cette fédéralisation est devenue d'une telle complexité - on parle depuis longtemps de "chirurgie institutionnelle» - qu'un plaidoyer subtil en faveur de sa "rationalisation ${ }^{21}$ » a valu aux séparatistes de la NieuwVlaamse Alliantie (Alliance néo-flamande) de devenir le premier parti de la région flamande en 2010 et, de facto, le premier parti du pays ${ }^{22}$.

L'autonomisme wallon se montre moins revendicatif que l'autonomisme flamand, dont il est globalement une réplique plus modérée et moins bien représentée dans l'espace politique. Mais étant l'un et l'autre issus de la matrice antinomique belge, ils ont en commun de combiner universalisme français et universalisme allemand dans une commune aspiration à un État-nation de type ethnique, c'est-à-dire linguistiquement et culturellement unifié. Cette antinomie politique en croise une autre, à dominante culturelle. En Flandre, l'impératif linguistique, le nomos «ici, on parle néerlandais», a toujours entretenu et continue d'entretenir une hésitation de type antinomique entre le

20. L'Accord de Versailles de 1919 a octroyé à la Belgique une dizaine de communes devenues prussiennes en 1814. Neuf d'entre elles ont été réunies dans la Communauté Germanophone de Belgique (Deutschsprachige Gemeinschaft Belgiens), qui a pour siège de gouvernement et de parlement Eupen et qui compte aujourd'hui 78.000 habitants dont la langue officielle est l'allemand. Très active sur les plans institutionnel et culturel, la plus petite des trois «communautés» belges rappelle que la "question linguistique» n'a pas en Belgique un caractère forcément conflictuel.

21. À travers un "confédéralisme» dont la médiatisation passe sous silence le fait qu'il impliquerait d'abord une mise à plat séparatiste (Paul DIRKX, «L'autre laboratoire européen du séparatisme. En Belgique, le poker menteur comme méthode de gouvernement", Le Monde diplomatique, novembre 2017, p. 10-11).

22. Tous les partis de gouvernement nationaux ont été scindés après le début $\mathrm{du}$ processus de fédéralisation en 1970 . 
néerlandais belge (souvent qualifié de "flamand») et le néerlandais des Pays-Bas. En Wallonie et à Bruxelles, une antinomie homologue entre le français belge et le français hexagonal travaille également les usages, mais elle penche plus nettement en faveur de sa composante hétéronome du fait d'une identification plus massive à la norme hexagonale perçue comme "universelle» (le wallon, quant à lui, est devenu aujourd'hui tout à fait minoritaire).

On voit ainsi que la complexité des phénomènes linguistiques en Belgique nécessite une approche systémique (macrostructurelle) et différentielle (microstructurelle), à laquelle le concept d'antinomie peut contribuer en corrélant les langues, leurs locuteurs et leurs croyances sociopolitiques. La dialectique des identifications linguistiques ne peut être séparée de l'antinomie politique qui caractérise l'État belge et que celui-ci n'a pas su convertir durablement en argument pro domo. Il en va de même pour les pratiques littéraires et les investissements «identitaires» qui les sous-tendent.

\section{Effets de l'antinomie politique sur les pratiques littéraires}

Sous l'effet, entre autres, du mécontentement au sujet de la minorisation de la langue néerlandaise, les institutions culturelles ont joué un rôle important dans la dualisation de la société belge. Dès 1886, l'État fonde une Koninklijke Academie voor Vlaamse Taal- en Letterkunde (Académie royale de linguistique et de littérature flamandes), rebaptisée en 1974 Koninklijke Academie voor Nederlandse Taal- en Letterkunde (Académie royale de linguistique et de littérature néerlandaises). En 1921, cette institution reçoit un pendant francophone, l'Académie royale de langue et de littérature françaises. Mais, à l'instar des langues, les deux littératures concernées ont chacune à se déployer à l'ombre de traditions nationales affirmées aux Pays-Bas et surtout en France, où la littérature est depuis plusieurs siècles le parangon de l'expansionnisme linguistico-littéraire le plus ambitieux du continent. Les activités littéraires se voient ainsi structurées, d'une part, par des rapports de force inhérents à l'organisation étatique belge qui sont peu favorables à l'avènement d'une littérature unique, nationale ; d'autre part, par des rapports de force spécifiquement linguistico-littéraires peu propices à une entité littéraire «flamande» ou «française» autonome. 
Le projet d'une littérature belge en langue française (le cas néerlandophone est largement comparable) a donc tout de la quadrature du cercle. Si une littérature nationale se développa au cours des premières décennies de l'État-nation, elle ne résista pas longtemps à l'injonction de monolinguisme inscrite dans le paradigme franco-universaliste. Cette même exigence inconditionnelle conduisit ensuite les milieux littéraires francophones à se diviser sur le caractère "vraiment» belge de leur littérature écrite en langue "française» et à remplacer peu à peu, au fil du XX siècle, l'appellation «littérature belge de langue française» par «littérature française de Belgique». À mesure que cette dénationalisation se confirmait, un nombre grandissant d'écrivains tendaient à souscrire aux modèles d'écriture conformes au nomos littéraire français réputé universel, "écrire» (sous-entendu : en tant qu'écrivain français). En revanche, les modèles liés davantage au nomos littéraire local, "écrire en tant qu'écrivain belge», se voyaient toujours plus associés à une futilité régionaliste ou formaliste et toujours moins pris au sérieux, y compris par ceux qui les pratiquaient (par exemple, certains auteurs "paralittéraires" de romans policiers ou fantastiques ou certains héritiers du dadaïsme et du surréalisme), s'ils n'étaient pas délaissés pour des formes "non » littéraires, telles la chanson ou la bande dessinée.

La littérature étant le secteur où la triade langue-culture-nation opère de la manière la plus flagrante quoique, à la fois, la plus déniée, l'approche comparatiste et sociale des littératures belges et d'autres "petites» littératures au profil multiculturel similaire a beaucoup contribué à mieux appréhender les rapports de force en question. Cette approche considère généralement que les deux littératures ont un statut "périphérique» ou "dominé" par rapport à leurs homologues "centrales» ou «dominantes ${ }^{23}$ ». Or ces couples oppositionnels incitent

23. Sur la situation de la littérature germanophone dans l'est du pays, voir Arvi SEPP, "Mémoire et minorité : l'identité collective dans la littérature germanophone de Belgique", Recherches germaniques, n 46, 2016, URL : http://journals.openedition. $\mathrm{org} / \mathrm{rg} / 323$ (consulté le 26 septembre 2019). Pour des éléments sur la question, encore relativement peu étudiée, des rapports entre la situation linguistique globale et les productions littéraires en Belgique, on lira Pierre BOURDIEU, "Existe-t-il une littérature belge ? Limites d'un champ et frontières politiques", Etudes de lettres, $\mathrm{n}^{\circ}$ 4, 1985, p. 3-6 ; Jean Weisgerber (dir.), Les avant-gardes littéraires en Belgique. Au 
plus d'un chercheur à avoir une vision relativement schématique des choses. Cela n'est pas dû à l'opposition centre / périphérie en tant que telle, proposée dans les années 1920 par les formalistes russes et élaborée un demi-siècle plus tard par les théoriciens du polysystème notamment ${ }^{24}$, ni, encore moins, au couple dominant / dominé ${ }^{25}$ : toutes ces notions concourent indéniablement à une description plus cohérente des structures, des institutions et des textes littéraires en Belgique. Le problème vient de ce qu'elles négligent un troisième terme, lié à ce que Max Weber décrivait comme étant la participation des dominés à leur propre domination à travers la reconnaissance de la légitimité des dominants ${ }^{26}$. Cette "complicité objective des dominés", pour parler avec Bourdieu ${ }^{27}$, ne repose pas sur une conscience claire de leur situation, mais sur l'harmonie préétablie entre leurs schèmes de vision et d'action (habitus) et les structures de la domination, fruit d'un processus d'incorporation préalable de ces dernières. En un mot, le point aveugle de la plupart des analyses des faits littéraires "périphériques", "dominés», "mineurs» ou encore "subalternes» nous paraît être la corporéité des agents et leur perméabilité en tant qu'êtres incorporants. Ce que les schémas duels font souvent perdre de vue, c'est le corps, non seulement comme incarnation de la position de tel écrivain dans tel champ littéraire, mais aussi et surtout en tant que réceptacle préalable des critères d'évaluation ("valeurs») de modèles littéraires plus ou moins

confluent des arts et des langues (1880-1950), Bruxelles, Labor, coll. Archives du futur, 1991 ; Marc QUAGHEBEUR, "L’identité ne se réduit pas à la langue», dans P. Gorceix (dir.), L'identité culturelle de la Belgique et de la Suisse francophones, Paris, Honoré Champion, coll. "Travaux et recherches des universités rhénanes», 1997, p. 59-105.

24. Iouri TyNIANOV, "Le fait littéraire» [1924], Formalisme et histoire littéraire. Traduit du russe, annoté et présenté par Catherine Depretto-Genty, L’Âge d'Homme, Lausanne, coll. "Slavica», 1991, p. 212-231 ; Itamar EvEN-ZOHAR, Papers in Historical Poetics, Tel Aviv, The Porter Institute for Poetics and Semiotics, 1978. En Belgique, le principal disciple d'Even-Zohar est José Lambert.

25. Sur la plus-value heuristique de dominant / dominé par rapport à centre / périphérie dans l'étude des systèmes littéraires (belges), voir Paul DIRKX, "Une périphérie ?», Christian Berg et Pierre Halen (dir.), Littératures belges de langue française (18302000). Histoire et perspectives, Bruxelles, Le Cri, 2000, p. 341-368.

26. M. WEBER, La domination, op. cit., p. 49 sq.

27. Pierre Bourdieu, Raisons pratiques. Sur la théorie de l'action, Paris, Seuil, 1994, p. 187. 
légitimes que cet écrivain a appris par corps depuis le début de sa prime socialisation littéraire (ou prime littérarisation) ${ }^{28}$.

La prise en compte de la corporéité de l'écrivain permet de penser la cohabitation inconciliable en son corps littérarisé des deux nomos littéraires, français et belge, dont l'un a plus de pouvoir définitionnel, plus de poids cognitif et social, est susceptible de procurer plus de capital symbolique littéraire, bref domine l'autre. Cette antinomie où les nomos sont du même ordre, en l'occurrence littéraire, peut être appelée "homogène». C'est elle qui, très diversement, soumet les corps d'écrivain "périphériques" à des hésitations ou des tiraillements qui tendent à les orienter vers le nomos dominant - souvent littéralement, physiquement, en les portant à partir pour la France et Paris -, et ce du fait que, pour en avoir incorporé les déterminants, ils y adhèrent malgré eux, par "soumission doxique ${ }^{29}$ ", c'est-à-dire, le corps étant une entité non pas simplement physiologique mais symbolico-physique ${ }^{30}$, par soumission corporelle, "naturellement». C'est l'antinomie homogène qui est au fondement de cette sorte de fragilité que l'écrivain francophone belge (ou suisse, canadien, africain, etc.) vit dans son corps socialisé littérairement (littérarisé) et qui fait régner à tout moment sur son écriture un doute sur sa propre légitimité ou tout au moins une insatisfaction, pénibles et en même temps souvent productifs ${ }^{31}$ : une insécurité littéraire.

Mais l'antinomie homogène croise aussi une antinomie plus ordinaire, hétérogène, où le nomos littéraire se confronte à d'autres nomos, politique, économique, éthique, etc. Tout écrivain, quel qu'il soit, a affaire à des appels de pied d'ordre politique, économique, etc. plus ou moins équilibrés par des rappels à l'ordre littéraire. L'écrivain belge de langue française a ceci de particulier qu'il importe en outre dans ses pratiques d'écriture, de manière plus ou moins impensée, des préférences politiques plutôt «françaises» ou plutôt «belges» (et,

\footnotetext{
28. Sur la socialisation comme apprentissage par corps, voir Pierre BOURDIEU, Le sens pratique, Paris, Minuit, coll. «Le sens commun», 1980, p. 123 et passim.

29. Ibid., p. 126-127.

30. Cf. Norbert EliAS, Théorie des symboles. Traduit de l'anglais par DamienGuillaume et Marie-Blanche Audollent. Révisé, édité et présenté par Marc Joly, Paris, Seuil, coll. «La librairie du XX ${ }^{e}$ siècle», 2015 [1991].

31. Lire les travaux de Marc Quaghebeur sur les «irréguliers du langage».
} 
de plus en plus, "wallonnes»), c'est-à-dire une combinaison des deux. Souvent, il nie la possibilité d'une littérature belge viable, se voyant en militant individuel de la cause littéraire française "universelle» au même titre que ses confrères français, suisses ou canadiens, tels les vingt et un signataires du manifeste du Groupe du Lundi de mars 1937, dont les positions devinrent hégémoniques dans les quatre décennies suivant la Seconde Guerre mondiale. Parfois, particulièrement enclin à méconnaître la réalité de sa littérarisation, il prétend à une écriture dénuée de la moindre spécificité "belge», une écriture tout entière au service de la littérature française - on pense par exemple à Charles Plisnier, le premier lauréat «français» non français du Prix Goncourt en 1937. De moins en moins rares sans doute sont, à l'inverse, les auteurs qui, comme par exemple le poète Christian Dotremont, entendent combattre l'antinomie indistinctement hétérogène et homogène inscrite dans l'expansionnisme littéraire français et vécue par eux comme un obstacle à leur autonomie d'écrivains nés et socialisés à un endroit du monde appelé Belgique.

Dans chaque cas de figure, un mélange antinomique aussi complexe qu'irréductible donne à l'écriture une tonalité à nulle autre pareille, souvent grinçante ou hilarante, et fondamentalement marquée par les catégories antinomiques d'un État déroutant - déroutant si l'on se place du point de vue de l'État français et de son fantasme triadique, que l'État belge et ses deux avatars en devenir ont par trop incorporé. 